\title{
REDUCTION OF LOCAL LIE ALGEBRA STRUCTURES
}

\author{
KENTARO MIKAMI
}

(Communicated by Jonathan Rosenberg)

\begin{abstract}
We deal with the reduction theory of local Lie algebra structures in the function space through coadjoint equivariant momentum mappings.
\end{abstract}

The Marsden-Weinstein symplectic reduction theory through momentum mappings [4] seems to be an epoch-making and remarkable theory in symplectic geometry. There are too many works which have applied that theory to refer to them all here. Marsden and Ratiu [3] gave a generalization of MarsdenWeinstein reduction theory in the Poisson manifold case. Also, Albert [1] has generalized Marsden-Weinstein reduction theory in the contact manifold case.

A local Lie algebra structure [2] in a function space is a more general idea than that of symplectic, Poisson or contact structure. It is also called a Jacobi structure by Lichnerowicz and coworkers. Since a Poisson manifold is a union of symplectic manifolds which fit together in a smooth way [6], it can be expected in some cases that a Poisson manifold divided by some relation or symmetry is a symplectic manifold. In the local Lie algebra structure case, it is natural to ask if a local Lie algebra structure divided by some relation is again a local Lie algebra, Poisson, or symplectic structure. In this note, we shall study reduction theory through momentum mappings of local Lie algebra structures.

Let $P$ be a $C^{\infty}$-manifold. A local Lie algebra structure in $C^{\infty}(P)$ is a bracket operation $\{$,$\} in C^{\infty}(P)$ satisfying:

(1) $\{$,$\} defines a Lie algebra structure in C^{\infty}(P)$ over $\mathbf{R}$.

(2) $\{f, h\}$ is continuous both in $f$ and $h$ with respect to the $C^{\infty}$-topology, and

Received by the editors July 17,1987 .

1980 Mathematics Subject Classification (1985 Revision). Primary 53C15; Secondary 58F05.

Key words and phrases. Local Lie algebra structure, Jacobi structure, momentum mapping, reduction.

This work was partially supported by Grant-in-Aid for Scientific Research No. 62540009, Ministry of Education, Science and Culture, Japan. 
(3) $\operatorname{supp}\{f, h\} \subset \operatorname{supp} f \cap \operatorname{supp} h$ for each $f$ and $h$, where $\operatorname{supp} f=$ the support of a function $f$ (cf. [2]).

It is shown in [2] that each local Lie algebra structure in $C^{\infty}(P)$ corresponds to a unique pair $(\xi, \Xi)$ consisting of a vector field $\xi$ and a 2 -vector field $\Xi$ on $P$ as follows:

$$
\begin{aligned}
\{f, h\} & =f(\xi\rfloor d h)-h(\xi\rfloor d f)+\langle\Xi \mid d f \wedge d h\rangle \\
& =f[\xi, h]-h[\xi, f]-[[\Xi, f], h],
\end{aligned}
$$

where $[$,$] denotes the Schouten bracket (cf. [5]). \xi$ and $\Xi$ must satisfy

$$
[\xi, \Xi]\left(=\mathscr{L}_{\xi} \Xi\right)=0 \text { and }[\Xi, \Xi]+2 \xi \wedge \Xi=0 \text {. }
$$

Remarks (cf. [2]). (1) If $\xi=0$, the local Lie algebra structure is a Poisson structure.

(2) If $\xi=0$ and $\operatorname{rank} \Xi=\operatorname{dim} P$, the local Lie algebra structure is the Poisson structure induced from the symplectic structure $\Xi^{-1}$.

(3) If $\xi$ and $\left\{[f, \Xi] \mid f \in C^{\infty}(P)\right\}$ span the tangent space of an odd-dimensional manifold $P$ at each point, then $\{$,$\} is the Lagrange bracket of a contact$ structure.

As an analogy of the Poisson reduction theorem [3], we would like to consider the reduction problem for local Lie algebra structures. Let $i: M \subset P$ be a submanifold of $P$. Let $\mathscr{E}$ be a subbundle of $T P_{\mid M}$. Assume that

(1) $\mathscr{E} \cap T M$ is an integrable subbundle of $T P_{\mid M}$,

(2) the foliation $\Phi$ corresponding to $\mathscr{E} \cap T M$ is regular, that is, $M / \Phi$ is a $C^{\infty}$-manifold and the canonical projection $\pi: M \rightarrow M / \Phi$ is a $C^{\infty}$-submersion, and

(3) $\left[\xi, \mathscr{E}^{0}\right] \subset \mathscr{E}^{0}$ and $\left[\left[\Xi, \mathscr{E}^{0}\right], \mathscr{E}^{0}\right] \subset \mathscr{E}^{0}$, where $\mathscr{E}^{0}:=\left\{F \in C^{\infty}(P)|v| d F\right.$ $=0$ for each $v \in \mathscr{E}$ \}.

Borrowing from Marsden-Ratiu's work, we say that $(P, M, \mathscr{E})$ is reducible if $M / \Phi$ has a local Lie algebra structure $\{,\}_{\Phi}$ such that for any $C^{\infty}$-functions $f$ and $h$ on $M / \Phi$ and smooth extensions $F$ and $H$ of $f \circ \pi$ and $h \circ \pi$ with $F, H \in \mathscr{E}^{0}$, we have $\{F, H\} \circ i=\{f, h\}_{\Phi} \circ \pi$.

Proposition. $(P, M, \mathscr{E})$ is reducible if and only if $\left[\xi,(\mathscr{E}+T M)^{0}\right]=0$ and $\left[\left[\Xi, \mathscr{E}^{0}\right],(\mathscr{E}+T M)^{0}\right]=0$ on $M$.

Proof. Take $F \in(\mathscr{E}+T M)^{0}$. Then there is a constant function, say $c$, on $M / \Phi$ such that $c \circ \pi=F \circ i$. We get

$$
\begin{aligned}
0 & =\{1, c\}_{\Phi} \circ \pi=\{1, F\} \circ i \\
& =([\xi, F]-[[\Xi, 1], F]) \circ i=[\xi, F] \circ i .
\end{aligned}
$$


Take $H \in \mathscr{C}^{0}$. Then there is a function $h$ such that $H \circ i=h \circ \pi$. Let $F_{0}$ be $F-c$. Then $F_{0} \in(\mathscr{E}+T M)^{0}$ and $F_{0} \circ i=0$. Now, we have that

$$
\begin{aligned}
\{F, H\} \circ i & =\left\{F_{0}+c, H\right\} \circ i=\left\{F_{0}, H\right\} \circ i+\{c, H\} \circ i \\
& =\left\{F_{0}, H\right\} \circ i+\{c, h\}_{\Phi} \circ \pi .
\end{aligned}
$$

Since $\{F, H\} \circ i=\{c, h\}_{\Phi} \circ \pi$, we get that $\left\{F_{0}, H\right\} \circ i=0$. So, it holds that

$$
\begin{aligned}
0= & \left\{F_{0}, H\right\} \circ i \\
= & \left(F_{0}[\xi, H]-H\left[\xi, F_{0}\right]-\left[\left[\Xi, F_{0}\right], H\right]\right) \circ i \\
= & \left(F_{0} \circ i\right)[\xi, H] \circ i-(H \circ i)\left[\xi, F_{0}\right] \circ i \\
& -\left(\left[\left[\Xi, F_{0}\right], H\right]\right) \circ i \\
= & -\left(\left[\left[\Xi, F_{0}\right], H\right]\right) \circ i .
\end{aligned}
$$

Conversely, for $f, h \in C^{\infty}(M / \Phi)$, take $F, H \in \mathscr{E}^{0}$ such that $F \circ i=f \circ \pi$ and $H \circ i=h \circ \pi$. We have that

$$
\begin{aligned}
{[\mathscr{E},\{F, H\}]=} & {[\mathscr{E}, F][\xi, H]+F[\mathscr{E},[\xi, H]] } \\
& -[\mathscr{E}, H][\xi, F]-H[\mathscr{E},[\xi, F]] \\
& -[\mathscr{E},[[\Xi, F], H]] \\
= & 0
\end{aligned}
$$

from assumption (3). This means that $\{F, H\}$ is constant on leaves of $\Phi$. So, define $\{f, h\}_{\Phi}$ by $\{f, h\}_{\Phi} \circ \pi:=\{F, H\} \circ i$. To check that $\{,\}_{\Phi}$ is well defined, take $H^{\prime} \in \mathscr{E}^{0}$ such that $H^{\prime} \circ i=h \circ \pi$. Then $H-H^{\prime} \in(\mathscr{E}+T M)^{0}$ and $\left(H-H^{\prime}\right) \circ i=0$. Therefore, we have

$$
\begin{aligned}
\left\{F, H-H^{\prime}\right\} \circ i= & F \circ i\left[\xi, H-H^{\prime}\right] \circ i \\
& -\left(H-H^{\prime}\right) \circ i[\xi, F] \circ i \\
& -\left[[\Xi, F], H-H^{\prime}\right] \circ i \\
= & 0 .
\end{aligned}
$$

It is easy to check skew-symmetry, Jacobi's identity and locality of $\{,\}_{\Phi}$.

\section{4}

By analogy to Hamiltonian vector fields on symplectic manifolds or infinitesimal contact transformations on contact manifolds, we consider a linear map $\beta: C^{\infty}(P) \rightarrow \mathscr{X}(P)$ defined by

$$
\beta(f):=f \xi-[f, \Xi] .
$$

$\beta$ is a Lie algebra homomorphism of $C^{\infty}(P)$ into $\mathscr{X}(P)$. So, we can now consider momentum mappings for a local Lie algebra structure. 
Definition [5]. Let a Lie group $G$ act on $P$ and preserve the local Lie algebra structure which is defined by $(\xi, \Xi)$. A map $J: P \rightarrow \mathfrak{g}^{*}$ is a momentum mapping of the action of $G$ if $(\beta \circ \widehat{J})(\zeta)=-\zeta_{P}$ for each $\zeta \in \mathfrak{g}$, where $\zeta_{P}$ is the fundamental vector field on $P$ defined by the action of $G$ and $\widehat{J}: \mathfrak{g} \rightarrow C^{\infty}(P)$ is defined by $\widehat{J}(\zeta)(x)=\langle\zeta, J(x)\rangle$ and is called the dual of $J$ or the co-momentum mapping of $J$.

The importance of momentum mappings in symplectic or Poisson geometry seems to stand on Noether's theorem. In our local Lie algebra structure case, we have

Noether's theorem [5]. Let $f$ be a G-invariant function on $P$. Then $\widehat{J}(\zeta)$ is a first integral of $\beta(f)$ for each $\zeta \in \mathfrak{g}$ if $f$ is invariant by $\xi$.

Now we shall study local Lie algebra structure reduction through an equivariant momentum mapping. Concerning the equivariance of momentum mappings of local Lie algebra structures, we refer to [5]. Let $J: P \rightarrow \mathfrak{g}^{*}$ be a coadjoint equivariant momentum mapping, and let $\mu \in \mathfrak{g}^{*}$ be a clean value of $J$, i.e., $\mu$ is a regular value and $T_{x} J^{-1}(\mu)=\operatorname{Ker} T_{x} J$ for each $x \in J^{-1}(\mu)$. Let $\mathscr{E}$ be the collection of tangent vectors to the $G$-orbits and let $M$ be $J^{-1}(\mu)$. Assume that

(1) $\mathscr{E} \cap T M=\left\{\eta_{P}(x) \mid x \in M, \eta \in \mathfrak{g}_{\mu}\right\}$ is a subbundle of $T P_{\mid M}$.

(2) The foliation $\Phi$ corresponding to $\mathscr{E} \cap T M$ is regular.

It is true that $\mathscr{E} \cap T M$ is integrable, $\left[\xi, \mathscr{E}^{0}\right] \subset \mathscr{E}^{0}$, and $\left[\left[\Xi, \mathscr{E}^{0}\right], \mathscr{E}^{0}\right] \subset \mathscr{E}^{0}$. Then we have the following theorem.

Theorem. Under the assumptions above, $\left(P, J^{-1}(0), \mathscr{E}\right)$ is reducible.

Lemma. (1) $\xi$ is tangent to $M=J^{-1}(\mu)$, and

(2) $\left[\xi,(\mathscr{E}+T M)^{0}\right]=0$ on $M$.

Proof. To show $T J(\xi)=0$, we deal with $\langle T J(\xi), \zeta\rangle=[\xi, \widehat{J}(\zeta)]$ for $\zeta \in \mathfrak{g}$. Since $-\zeta_{P}=\beta \widehat{J}(\zeta)=\widehat{J}(\zeta) \xi-[\widehat{J}(\zeta), \Xi]$ preserves the local Lie algebra structure, we have

$$
0=-\left[\zeta_{P}, \xi\right]=[\widehat{J}(\zeta), \xi] \xi-[[\widehat{J}(\zeta), \xi], \Xi]
$$

and

$$
0=-\left[\zeta_{P}, \Xi\right]=[\widehat{J}(\zeta), \xi] \Xi
$$

Fix an arbitrary point $x \in P$. If $\Xi_{x} \neq 0$, the second equation implies $[\widehat{J}(\zeta), \xi]_{x}=0$. If $\Xi_{x}=0$, then the first equation implies that $[\widehat{J}(\zeta), \xi]_{x} \xi_{x}=0$, and we have $[\widehat{J}(\zeta), \xi]_{x}=0$. Therefore, we have $[\widehat{J}(\zeta), \xi]=0$ for each $\zeta \in \mathfrak{g}$.

Proof of theorem. Take $F \in(\mathscr{E}+T M)^{0}$ and $H \in \mathscr{E}^{0}$. We have

$$
\begin{aligned}
\langle T J[\Xi, H], \zeta\rangle & =[[\Xi, H], \widehat{J}(\zeta)]=-[[\Xi, \widehat{J}(\zeta)], H] \\
& =[\beta \widehat{J}(\zeta)-\widehat{J}(\zeta) \xi, H]=\left[-\zeta_{P}, H\right]-\widehat{J}(\zeta)[\xi, H]=-\widehat{J}(\zeta)[\xi, H]
\end{aligned}
$$


for each $\zeta \in \mathfrak{g}$. If $\mu=0 \in \mathfrak{g}^{*}$, then $\widehat{J}(\zeta)=0$ on $M=J^{-1}(\mu)$ and the above implies that $T J[\Xi, H]=0$, that is, $[\Xi, H] \in T M$. Therefore, we have $[[\Xi, H], F]=0$ on $M$. Thus, the Lemma and Proposition complete the proof of our theorem.

Example. Let $P$ be $\mathbf{R}^{2 n}$ with the standard coordinates $\left(x^{1}, y^{1}, \ldots, x^{n}, y^{n}\right)$. Define a vector field $\xi$ and a 2 -vector field $\Xi$ on $P$ by

$$
\xi=\sum_{i}\left(x^{i} \frac{\partial}{\partial y^{i}}-y^{i} \frac{\partial}{\partial x^{i}}\right)
$$

and

$$
\begin{aligned}
2 \Xi= & +\sum_{i} x^{i} \frac{\partial}{\partial x^{i}} \wedge \sum_{j} y^{j} \frac{\partial}{\partial x^{j}}+\sum_{i} x^{i} \frac{\partial}{\partial y^{i}} \wedge \sum_{j} y^{j} \frac{\partial}{\partial y^{j}} \\
& -\sum_{i} x^{i} \frac{\partial}{\partial x^{i}} \wedge \sum_{j} x^{j} \frac{\partial}{\partial y^{j}}-\sum_{i} y^{i} \frac{\partial}{\partial x^{i}} \wedge \sum_{i} y^{j} \frac{\partial}{\partial y^{j}}+c \sum_{i} \frac{\partial}{\partial x^{i}} \wedge \frac{\partial}{\partial y^{i}},
\end{aligned}
$$

where $c$ is a constant number. Then $(\xi, \Xi)$ defines a local Lie algebra structure on $P=\mathbf{R}^{2 n}$.

(i) The abelian Lie group $\mathbf{R}$ whose Lie algebra is $\mathbf{R} \xi$ acts on $(P, \xi, \Xi)$. If $c \neq 0$, the action of $\mathbf{R}$ has a coadjoint equivariant momentum mapping

$$
J\left(x^{1}, y^{1}, \ldots, x^{n}, y^{n}\right)=\frac{1}{c} \sum_{i}\left(x^{i} x^{i}+y^{i} y^{i}\right) .
$$

But $J^{-1}(0)$ is the one point set $\{0\}$.

(ii) Let $G$ be the connected Lie subgroup of $\operatorname{GL}(2 n, \mathbf{R})$ whose Lie algebra is generated by

$$
\begin{aligned}
\zeta_{1}=\left[\begin{array}{rrr}
0 & I & 0 \\
-I & 0 & 0 \\
0 & 0 & 0
\end{array}\right], & \zeta_{2}=\left[\begin{array}{ccc}
0 & K & 0 \\
K & 0 & 0 \\
0 & 0 & 0
\end{array}\right], \\
\zeta_{3}=\left[\begin{array}{lll}
K & 0 & 0 \\
0 & 0 & 0 \\
0 & 0 & 0
\end{array}\right], & \zeta_{4}=\left[\begin{array}{lll}
0 & 0 & 0 \\
0 & K & 0 \\
0 & 0 & 0
\end{array}\right],
\end{aligned}
$$

where $I$ and $K$ are $2 \times 2$-matrices $I=\left[\begin{array}{ll}1 & 0 \\ 0 & 1\end{array}\right]$ and $K=\left[\begin{array}{cc}0 & 1 \\ -1 & 0\end{array}\right]$. Then $G$ preserves the local Lie algebra structure. If $c \neq 0$, the action of $G$ has a coadjoint equivariant momentum mapping $J$ defined by $\widehat{J}\left(\zeta_{K}\right)=F_{k}$, where

$$
\begin{array}{ll}
F_{1}=\frac{2}{c}\left(-x^{1} y^{2}+x^{2} y^{1}\right), & F_{2}=\frac{2}{c}\left(x^{1} x^{2}+y^{1} y^{2}\right), \\
F_{3}=\frac{1}{c}\left(x^{1} x^{1}+y^{1} y^{1}\right), & F_{4}=\frac{1}{c}\left(x^{2} x^{2}+y^{2} y^{2}\right) .
\end{array}
$$

It turns out that $J^{-1}(0)=\left\{\left(x^{1}, y^{1}, \ldots, x^{n}, y^{n}\right) \in \mathbf{R}^{2 n} \mid x^{1}=y^{1}=x^{2}=y^{2}=\right.$ $0\} \simeq \mathbf{R}^{2 n-4}, G$ acts trivially on $J^{-1}(0)$ and the reduced space is of dimension $2(n-2)$ and has a local Lie algebra structure of the same type as the original local Lie algebra structure of $P=\mathbf{R}^{2 n}$. 


\section{REFERENCES}

1. C. Albert, Le théorème de réduction de Marsden-Weinstein pour les structures de contact, Seminaire de Géom. et Topologie Différentielles, Univ. des Sci. et Tech. du Languedoc, 1985/1986, pp. 21-34.

2. A. A. Kirillov, Local Lie algebras, Uspekhi Mat. Nauk. 31 (1976), no. 4, 57-76; English transl., Russian Math. Surveys 31 (1976), no. 4, 55-75.

3. J. Marsden and T. Ratiu, Reduction of Poisson manifolds, Lett. Math. Phys. 11 (1986), 161169.

4. J. Marsden and A. Weinstein, Reduction of symplectic manifolds with symmetry, Rep. Math. Phys. 5 (1974), 121-129.

5. K. Mikami, Local Lie algebra structure and momentum mapping, J. Math. Soc. Japan 39 (1987), 233-246.

6. A. Weinstein, The local structure of Poisson manifolds, J. Differential Geom. 18 (1983), 523557.

Department of Mathematics, Akita University, Akita 010, Japan 